\title{
Low-energy Quantum Gravity and Cosmology without Dark Energy
}

\author{
Michael A. Ivanov \\ Physics Dept., \\ Belarus State University of Informatics and Radioelectronics, \\ Minsk, Republic of Belarus
}

\begin{abstract}
The model of low-energy quantum gravity leads to small additional effects having essential cosmological consequences: redshifts of remote objects and the additional dimming of them may be interpreted without any expansion of the Universe and without dark energy. The theoretical luminosity distance of the model fits the observational Hubble diagrams with high confidence levels. In the model, the ratio $H(z) /(1+z)$ should be equal to the Hubble constant. The constancy of this ratio is confirmed with high probabilities by fitting the compilation of $H(z)$ observations. A deceleration of massive bodies due to forehead and backhead collisions with gravitons is re-computed here.
\end{abstract}

Keywords: Low-energy quantum gravity, graviton background, dark energy.

\section{Introduction}

In my model of low-energy quantum gravity $[1,2]$, gravity is considered as the screening effect. It is suggested that the background of super-strong interacting gravitons exists in the universe. Its temperature should be equal to the one of the CMB. Screening this background creates for any pair of bodies both attraction and repulsion forces due to pressure of gravitons. For single gravitons, these forces are approximately balanced, but each of them is much bigger than a force of Newtonian attraction. If single gravitons are pairing, an attraction force due to pressure of such graviton pairs is twice exceeding a corresponding repulsion force if graviton pairs are destructed by collisions with a body. This peculiarity of the quantum mechanism of gravity leads to the difference of inertial and gravitational masses of a black hole. In such a model, the Newton constant is connected with the Hubble constant that gives a possibility to obtain a theoretical estimate of the last. We deal here with a flat non-expanding universe fulfilled with super-strong interacting gravitons; it changes the meaning of the Hubble constant which describes magnitudes of three small effects of quantum gravity but not any expansion or an age of the universe.

In this model, the geometrical distance/redshift relation is:

$$
r(z)=\ln (1+z) \cdot c / H_{0},
$$

where $H_{0}$ is the Hubble constant, $c$ is the velocity of light, $z$ is a redshift. The luminosity distance/redshift relation has the view:

$$
D_{L}(z)=c / H_{0} \cdot \ln (1+z) \cdot(1+z)^{(1+b) / 2} \equiv c / H_{0} \cdot f_{1}(z),
$$

where $f_{1}(z) \equiv \ln (1+z) \cdot(1+z)^{(1+b) / 2}$; the "constant" $b$ belongs to the range $0-2.137(b=2.137$ for very soft radiation, and $b \rightarrow 0$ for very hard one).

\section{Deceleration of Massive Bodies Due to Forehead and Backhead Collisions with Gravitons}

Due to forehead collisions of a massive body with gravitons, the body acceleration $w$ by a non-zero velocity $v$ had been found [1] to be equal to:

$$
w=-c H_{0}\left(1-v^{2} / c^{2}\right) .
$$


But recently it was shown in [3] that this value is too large to provide, for example, the observed stability of the Earth-like orbit. Here I would like to re-analyze this problem.

The dependencies (1) and (3) have been gotten starting from the equation:

$$
d E=-\left(H_{0} / c\right) E d r
$$

describing average energy losses of a photon (or a body, as it was supposed in [1]) with an energy $E$ on a way $d r$. While for a photon its momentum $p$ and energy $E$ are proportional, for massive bodies it is not so. A transferred quantity by collisions is the momentum, and we should express its differential $d p$ before

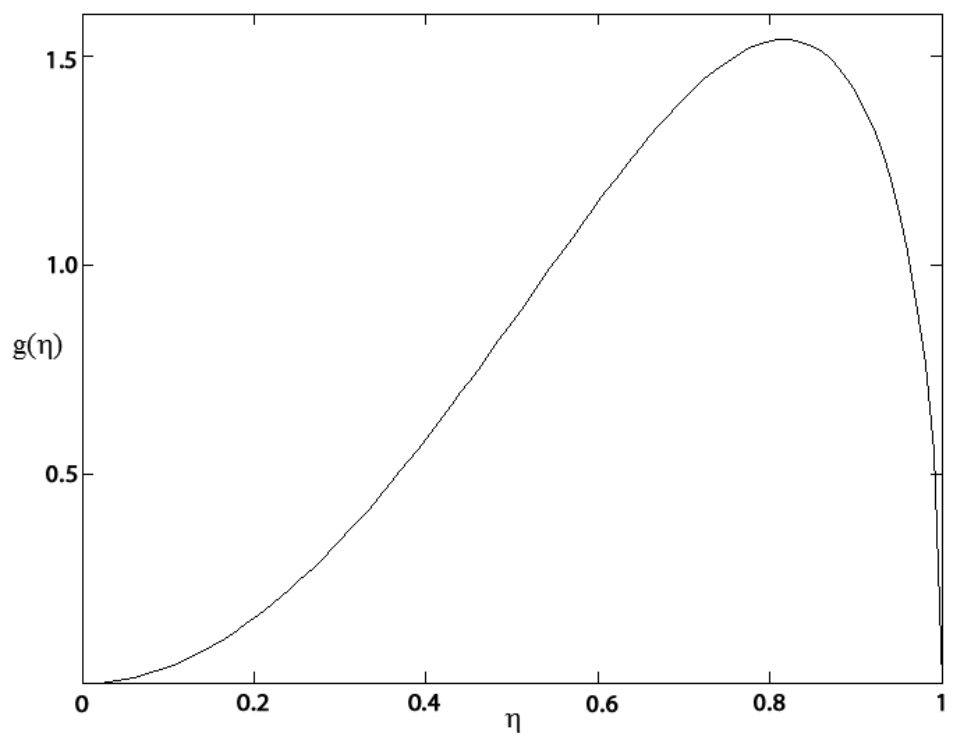

Figure 1. The graph of the function $g(\eta)$.

calculations of the body deceleration:

$$
d p=-\left(H_{0} / c^{2}\right) E d r .
$$

Besides of forehead collisions, the body should also experience backhead collisions with gravitons; it means that for massive bodies we can write the following similar expression:

$$
d p=-\left(H_{0 f} / c^{2}-H_{0 b} / c^{2}\right) E d r,
$$

where $H_{0 f}$ and $H_{0 b}$ correspond to forehead and backhead collisions with gravitons. This equation is written in the $\mathrm{CMB}$ frame $K$, in which the $\mathrm{CMB}$ is isotropic - in the sense that deviations from the isotropy cannot be made smaller in any other frame. We shall use here also the rest frame of the body $K^{\prime}$, which moves relatively to $K$ with the velocity $v$.

The Doppler effect should lead to the different values of energies of gravitons which are incident from the front and from the back in $K^{\prime}$. We can find the difference of $H_{0 f}^{\prime}$ and $H_{0 b}^{\prime}$ in $K^{\prime}$ and re-calculate it for $K$. So as $H_{0 f}, H_{0 b}$ and $H_{0 f}^{\prime}, H_{0 b}^{\prime}$ have the same dimensions as $\Delta t^{-1}$ and $\Delta t^{\prime}-1$, where $\Delta t$ and $\Delta t^{\prime}$ are the time intervals between two events in these frames, we have:

$$
H_{0 f}-H_{0 b}=\left(H_{0 f}^{\prime}-H_{0 b}^{\prime}\right) \cdot\left(1-\eta^{2}\right)^{0.5},
$$

where $\eta \equiv v / c$.

Because the number of gravitons (per unit of surface area per unit of time) falling on the body from the front or from the back is the same in $K^{\prime}$ as in $K$, their spectrum $f_{1}(\epsilon)$ in $K^{\prime}$ may be presented as:

$$
f_{1}(\epsilon)=f(\epsilon / \kappa, T)=\left(1 / \kappa^{3}\right) \cdot f(\epsilon, \kappa T),
$$


where $f(\epsilon, T)$ is the Planck spectrum in $K$ by the temperature T, $\epsilon$ is the graviton energy; $\kappa \equiv$ $\epsilon^{\prime} / \epsilon$ is the ratio of new and old (in $K^{\prime}$ and $K$ ) graviton energies. This spectrum is a result of the stretching/compression of the Planck spectrum by the same temperature $T$ along the $\epsilon$ axis in $\kappa$ times. For gravitons which are incident from the front and from the back in $K^{\prime}$, we have:

$$
\kappa_{f}=\left(\frac{1+\eta}{1-\eta}\right)^{0.5}, \kappa_{b}=\left(\frac{1-\eta}{1+\eta}\right)^{0.5} .
$$

In this model, the Hubble constant is equal to:

$$
H_{0}=\frac{1}{2 \pi} \int_{0}^{\infty} \hbar \omega f(\omega, T) d \omega=\frac{1}{2 \pi} D \cdot \bar{\epsilon} \cdot\left(\sigma T^{4}\right),
$$

where $D$ is a constant, $\bar{\epsilon}$ is an average graviton energy, $\sigma$ is the Stephan- Boltzmann constant, and $\epsilon=\hbar \omega$. Replacing $f(\omega, T) \rightarrow f_{1}(\omega)$, we have: $\bar{\epsilon} \rightarrow \kappa \cdot \bar{\epsilon}, \sigma T^{4} \rightarrow \kappa \cdot \sigma T^{4}$. As a result we get:

$$
\begin{aligned}
H_{0 f}^{\prime} & =\kappa_{f}^{2} \cdot H_{0}=H_{0} \cdot(1+\eta / 1-\eta), \\
H_{0 b}^{\prime} & =\kappa_{b}^{2} \cdot H_{0}=H_{0} \cdot(1-\eta / 1+\eta) .
\end{aligned}
$$

Then we can rewrite Eq.(6) as:

$$
d p=-\left(H_{0} / c^{2}\right)\left(\kappa_{f}^{2}-\kappa_{b}^{2}\right) \cdot\left(1-\eta^{2}\right)^{0.5} E d r=-\left(H_{0} / c^{2}\right) \cdot 4 \eta\left(1-\eta^{2}\right)^{-0.5} E d r .
$$

Taking into account that by $\mathbf{v} \| \mathbf{w}$, where $\mathbf{w} \equiv d \mathbf{v} / d t, d p / d t$ is equal to:

$$
d p / d t=m w \cdot\left(1-\eta^{2}\right)^{-1.5}
$$

and $E=m c^{2} \cdot\left(1-\eta^{2}\right)^{-0.5}, d r=v d t$, we get finally for the deceleration $w$ :

$$
w=-w_{0} \cdot 4 \eta^{2} \cdot\left(1-\eta^{2}\right)^{0.5}
$$

where $w_{0} \equiv H_{0} c=6.419 \cdot 10^{-10} \mathrm{~m} / \mathrm{s}^{2}$, if we use the theoretical value of $H_{0}$ in the model. For small velocities we have now:

$$
w \simeq-w_{0} \cdot 4 \eta^{2}
$$

The function $g(\eta) \equiv 4 \eta^{2} \cdot\left(1-\eta^{2}\right)^{0.5}$ in Eq. (13) has the maximum value of 1.54 by $\eta=(2 / 3)^{0.5}=0.816$, i.e. the maximum deceleration is equal to: $|w|_{\max }=1.54 \cdot w_{0}$. The graph of this function is shown in Fig. 1. As it was shown in [3], by $|w| \sim 10^{-4} \cdot w_{0}$ the stability of the Earth-like orbit will be high enough. By $v=4 \cdot 10^{5} \mathrm{~m} / \mathrm{s}$ we have now: $w \simeq-7 \cdot 10^{-6} \cdot w_{0}$. The numerical calculations of $[3]$ with the new formula for $w$ will be repeated soon.

The mass discrepancy in spiral galaxies is observed at very low accelerations less than $\sim 10^{-10} \mathrm{~m} / \mathrm{s}^{2}$ [4], i.e. this boundary acceleration has almost the same order of magnitude as the maximum deceleration $|w|_{\text {max }} \sim 10^{-9} \mathrm{~m} / \mathrm{s}^{2}$ in the model. Now it is unclear may these quantities be connected between themselves or not.

\section{The Hubble Diagram of This Model}

To fit this model, observations should be corrected for no time dilation as: $\mu(z) \rightarrow \mu(z)+2.5 \cdot \lg (1+z)$, where $\lg x \equiv \log _{10} x$. In my paper [5], results of fitting the Hubble diagram for different data sets of remote objects with the model of low-energy quantum gravity are summarized in Table 1; its part is shown here. For best fitting values of $b$ in a case of 44 long GRBs, values of distance moduli are overestimated in both calibrations: on $\sim 0.225$ for the Amati calibration, and on $\sim 1.18$ for the Yonetoku calibration. It leads to the corresponding underestimation of the Hubble constant. 
Table 1. Results of fitting the Hubble diagram with the model of low-energy quantum gravity. The best fitting values of $b$ for 44 long GRBs are marked by the bold typeface.

\begin{tabular}{lrrrr}
\hline Data set & $b$ & $\chi^{2}$ & C.L., $\%$ & $<H_{0}> \pm \sigma_{0}$ \\
\hline SCP Union 2.1 [6] & 2.137 & 239.635 & 100 & $68.22 \pm 6.10$ \\
\hline JLA [7] & 2.365 & 30.71 & 43.03 & $69.54 \pm 1.58$ \\
\hline 109 long GRBs [8] & 2.137 & 70.39 & 99.81 & $66.71 \pm 8.45$ \\
\hline 44 long GRBs [9], & 2.137 & 40.585 & 57.66 & $69.73 \pm 37.23$ \\
the Amati calibration & $\mathbf{1 . 8 8 5}$ & 39.92 & 60.57 & $60.31 \pm 31.93$ \\
\hline 44 long GRBs [9], & 2.137 & 43.148 & 46.5 & $70.39 \pm 38.79$ \\
the Yonetoku calibration & $\mathbf{1 . 1 1}$ & 32.58 & 87.62 & $38.84 \pm 18.55$ \\
\hline quasars [10] & 2.137 & 23.378 & 13.73 & $69.53 \pm 10.87$ \\
\hline
\end{tabular}

\section{The Hubble Parameter $H(z)$ of This Model}

If the geometrical distance is described by $E q$. 1, for a remote region of the universe we may introduce the Hubble parameter $H(z)$ in the following manner:

$$
d z=H(z) \cdot \frac{d r}{c}
$$

to imitate the local Hubble law. Taking a derivative $\frac{d r}{d z}$, we get in this model for $H(z)$ :

$$
H(z)=H_{0} \cdot(1+z)
$$

It means that in the model:

$$
\frac{H(z)}{(1+z)}=H_{0}
$$

The last formula gives us a possibility to evaluate the Hubble constant using observed values of the Hubble parameter $H(z)$. The weighted average value of the Hubble constant may be calculated by the formula:

$$
<H_{0}>=\frac{\sum \frac{H\left(z_{i}\right)}{1+z_{i}} / \sigma_{i}^{2}}{\sum 1 / \sigma_{i}^{2}} .
$$

The weighted dispersion of the Hubble constant may be found with the same weights:

$$
\sigma_{0}^{2}=\frac{\sum\left(\frac{H\left(z_{i}\right)}{1+z_{i}}-<H_{0}>\right)^{2} / \sigma_{i}^{2}}{\sum 1 / \sigma_{i}^{2}} .
$$

The $\chi^{2}$ value is calculated as:

$$
\chi^{2}=\sum \frac{\left(\frac{H\left(z_{i}\right)}{1+z_{i}}-<H_{0}>\right)^{2}}{\sigma_{i}^{2}} .
$$

In [5], I have done these calculations for two data sets of $H(z)$. Here I repeat them for the bigger data set of 40 observations of $H(z)$ from the paper [11]. We have for this case:

$$
<H_{0}> \pm \sigma_{0}=(62.082 \pm 4.092) \mathrm{km} \mathrm{s}^{-1} \mathrm{Mpc}^{-1}
$$

The weighted average value of the Hubble constant with $\pm \sigma_{0}$ error bars are shown in Fig. 2 as horizontal lines; observed values of the ratio $H(z) /(1+z)$ with $\pm \sigma$ error bars are shown in Fig. 2, too (points). The value of $\chi^{2}$ in this case is equal to 10.69 . By 40 degrees of freedom of this data set, it means that the hypothesis described by Eq. (16) cannot be rejected with $99.9999 \%$ C.L.

I have used earlier the same values of dispersion for $H_{0}$ points as the ones of $H(z)$. Considering Eq. (17) as a base for indirect measurements of $H_{0}$, we get for the dispersion $\sigma_{0 i}^{2}$ of $H_{0}$ points:

$$
\sigma_{0 i}^{2}=\sigma_{i}^{2} /\left(1+z_{i}\right)^{2}
$$




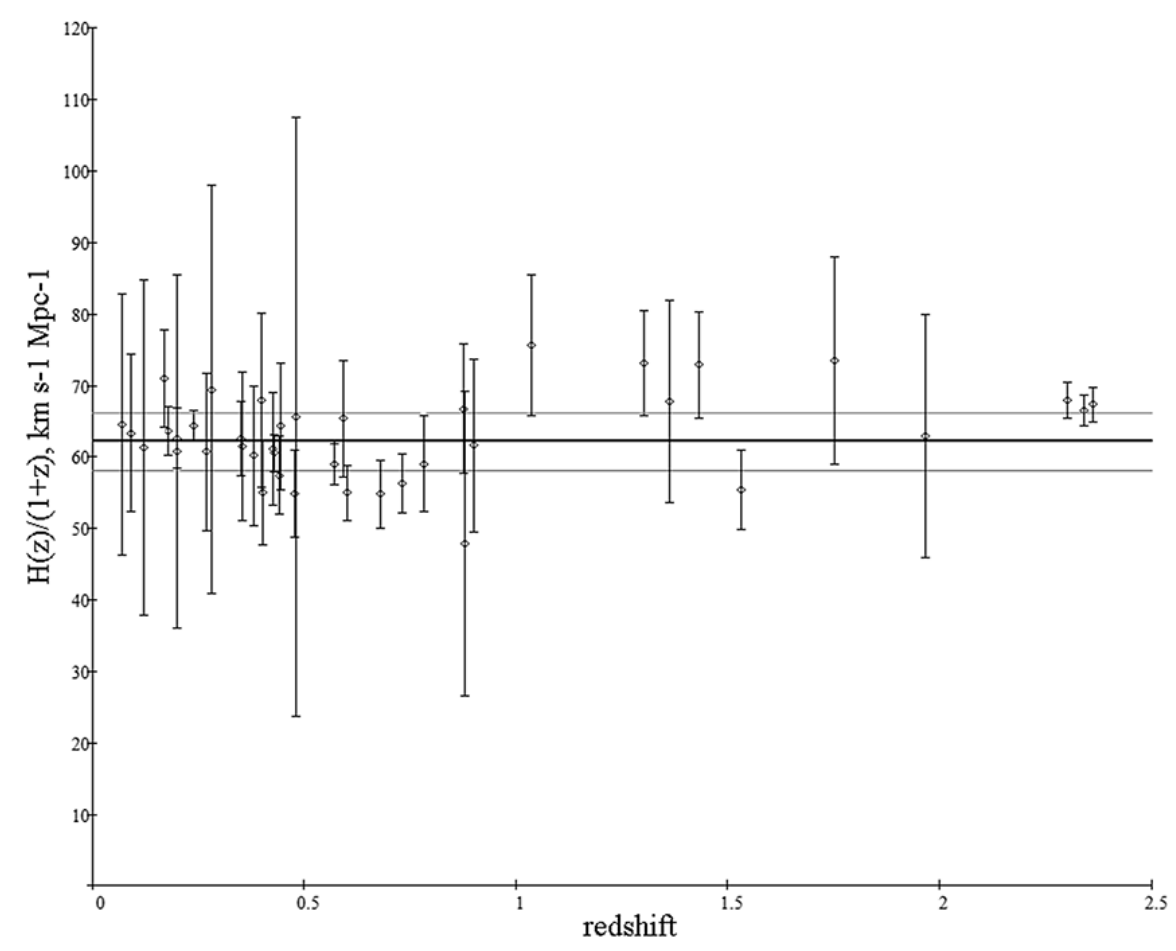

Figure 2. The ratio $H(z) /(1+z) \pm \sigma$ and the weighted value of the Hubble constant $\left\langle H_{0}\right\rangle \pm \sigma_{0}$ (horizontal lines). Observed values of the Hubble parameter $H(z)$ (40 points) are taken from Table 1 of [11].

Then we shall have for the considered data set:

$$
<H_{0}> \pm \sigma_{0}=(63.152 \pm 4.689) \mathrm{km} \mathrm{s}^{-1} \mathrm{Mpc}^{-1} .
$$

The value of $\chi^{2}$ is now equal to 38.56. By 40 degrees of freedom of this data set, it means that the hypothesis described by Eq. (16) cannot be rejected with $53.511 \%$ C.L. The dramatic increase of $\chi^{2}$ is mainly due to the last three points with $z>2$ in Fig. 2 with small $\sigma_{0 i}$ : without them, $\chi^{2}=24.857$ that gives $93.633 \%$ C.L. by 37 degrees of freedom.

The $R_{h}=c t$ cosmological model (a Friedmann-Robertson-Walker cosmology with zero active mass) has the same function $H(z)$ as the considered one [12]; $R_{h}$ is the Hubble radius. As it is shown in [12], this function fits 30 cosmic chronometers observations with $z<2$ with a larger probability than five other considered functions of different models, including the flat $\Lambda \mathrm{CDM}$.

Some authors try in a frame of models of expanding universe to find the deceleration-acceleration transition's redshift using the same data sets. The above conclusion that the ratio $H(z) /(1+z)$ remains statistically constant in the available range of redshifts is model-independent.

\section{Conclusion}

The Hubble diagram for GRBs may differ in the model from the diagram for SNe Ia, and some signs of this difference are seen, perhaps, in the case of the 44 long GRBs data set. In the model, space-time is flat, and the geometrical distance as a function of the redshift coincides with the angular diameter distance. The geometrical distance of this model is very different from the one of the standard model; for example, the age of the Universe of the standard model: $\sim 13.5 \mathrm{Gyr}$ corresponds here to $z \simeq 2.6$.

The found expression for the anomalous deceleration of massive bodies: $w=-w_{0} \cdot 4 \eta^{2} \cdot\left(1-\eta^{2}\right)^{0.5}$ should ensure a sufficient stability of the Earth-like orbits. It is planned to model numerically a modification of dynamics due to it very soon. 
From a point of view of this approach it seems that all attempts to unify general relativity and quantum mechanics using them as corner stones of the future more general theory are doomed to failure. The future theory should be underlaying one for general relativity and quantum mechanics which will be specific approximations of it by some restrictions.

\section{References}

1. M.A. Ivanov, "Gravitons as super-strong interacting particles, and low-energy quantum gravity", in Focus on Quantum Gravity Research, Ed. D.C. Moore. Nova Science, NY, 2006, pp. 89-120.

2. M.A. Ivanov, Selected papers on low-energy quantum gravity,2011. [Online]. Available: http://ivanovma.narod.ru/selected-papers-Ivanov11.pdf.

3. M.A. Ivanov, A.S. Narkevich, and P.S. Shenetz, "Modified dynamics due to forehead collisions of bodies with gravitons: Numerical modeling". [Online]. Available: http://vixra.org/pdf/1706.0427v1.pdf.

4. B. Famaey, and S. McGaugh, "Modified Newtonian Dynamics (MOND): Observational Phenomenology and Relativistic Extensions", Living Reviews in Relativity, vol. 15, p. 10, 2012.

5. M.A. Ivanov, "Cosmological consequences of the model of low-energy quantum gravity", in Cosmology on Small Scales 2016. Proceedings. M. Krizek and Yu. Dumin (Eds.), Institute of Mathematics CAS, Prague, pp. 179-198.

6. N. Suzuki et al, "The Hubble Space Telescope Cluster Supernova Survey: V. Improving the Dark Energy Constraints Above $z>1$ and Building an Early-Type-Hosted Supernova Sample", ApJ, vol. 746, p. 85, 2012.

7. M. Betoule et al, "Improved cosmological constraints from a joint analysis of the SDSS-II and SNLS supernova samples. [Online]. Available: arXiv:1401.4064v2 [astro-ph.CO].

8. H. Wei, Observational Constraints on Cosmological Models with the Updated Long Gamma-Ray Bursts". [Online]. Available: arXiv:1004.4951v3 [astro-ph.CO].

9. H.-N. Lin, X. Li, and Z. Chang, "Effect of GRB spectra on the empirical luminosity correlations and the GRB Hubble diagram". [Online]. Available: arXiv:1604.02285 [astro-ph.HE].

10. M. Lopez-Corredoira, F. Melia, E. Lusso, G. and Risaliti, "Cosmological test with the QSO Hubble diagram". [Online]. Available: arXiv:1602.06743 [astro-ph.CO].

11. M.-J. Zhang, and J.-Q. Xia, "Model independent analysis on the slowing down of cosmic acceleration". [Online]. Available: arXiv:1606.04398 [astro-ph.CO].

12. F. Melia, and M.K. Yennapureddy, "Model Selection Using Cosmic Chronometers with Gaussian Processes". [Online]. Available: arXiv:1802.02255 [astro-ph.CO]. 